\title{
BMJ Global Health Prevalence and incidence of HIV among female sex workers and their clients: modelling the potential effects of intervention in Rwanda
}

\author{
Sabin Nsanzimana, ${ }^{1,2}$ Edward J Mills (D) ,3,4 Ofir Harari, ${ }^{4}$ Placidie Mugwaneza, ${ }^{5}$ \\ Etienne Karita, ${ }^{6}$ Jean Paul Uwizihiwe, ${ }^{7,8}$ Jay JH Park, ${ }^{4,9}$ Louis Dron, ${ }^{4}$ \\ Jeanine Condo, ${ }^{10}$ Heiner Bucher, ${ }^{2}$ Kristian Thorlund ${ }^{3,4}$
}

\begin{abstract}
To cite: Nsanzimana S, Mills EJ, Harari 0, et al. Prevalence and incidence of HIV among female sex workers and their clients: modelling the potential effects of intervention in Rwanda. BMJ Global Health 2020;5:e002300. doi:10.1136/ bmjgh-2020-002300
\end{abstract}

\section{Handling editor Lei $\mathrm{Si}$}

- Additional material is published online only. To view please visit the journal online (http://dx.doi.org/10.1136/ bmjgh-2020-002300).

Received 7 January 2020 Revised 27 February 2020 Accepted 7 March 2020
Check for updates

(c) Author(s) (or their employer(s)) 2020. Re-use permitted under CC BY-NC. No commercial re-use. See rights and permissions. Published by BMJ.

For numbered affiliations see end of article.

Correspondence to Dr Sabin Nsanzimana; nsabinco@gmail.com

\section{ABSTRACT}

Background Rwanda has identified several targeted HIV prevention strategies, such as promotion of condom use and provision of antiretroviral therapy (ART) and pre-exposure prophylaxis (PrEP) for female sex workers (FSWs). Given this country's limited resources, understanding how the HIV epidemic will be affected by these strategies is crucial.

Methods We developed a Markov model to estimate the effects of targeted strategies to FSWs on the HIV prevalence/ incidence in Rwanda from 2017 to 2027. Our model consists of the six states: HIV-; HIV+ undiagnosed/diagnosed preART; HIV+ diagnosed with/without ART; and death. We considered three populations: FSWs, sex clients and the general population. For the period 2017-2027, the HIV epidemic among each of these population was estimated using Rwanda's demographic, sexual risk behaviour and HIVassociated morbidity and mortality data.

Results Between 2017 and 2027, with no changes in the current condom and ART use, the overall number of people living with HIV is expected to increase from 344,971 to 402,451 . HIV incidence will also decrease from 1.36 to 1.20100 person-years. By 2027, a 30\% improvement in consistent condom use among FSWs will result in absolute reduction of HIV prevalence among FSWs, sex clients and the general population by $7.86 \%, 5.97 \%$ and $0.17 \%$, respectively. While recurring HIV testing and improving the ART coverage mildly reduced the prevalence/incidence among FSWs and sex clients, worsening the two (shown by our worst-case scenario) will result in an increase in the HIV prevalence/incidence among FSWs and sex clients. Introduction of PrEP to FSWs in 2019 will reduce the HIV incidence among FSWs by $1.28 \%$.

Conclusions Continued efforts toward improving condom and ART use will be critical for Rwanda to continue their HIV epidemic control. Implementing a targeted intervention strategy in PrEP for FSWs will reduce the HIV epidemic in this high-risk population.

\section{INTRODUCTION}

Female sex workers (FSWs) are a key population in the sub-Saharan African HIV epidemic

\section{Key questions}

What is already known?

- Female sex workers (FSWs) are a key population that should be targeted for HIV prevention and treatment strategies. Improving consistent condom use, antiretroviral therapy (ART) and pre-exposure prophylaxis (PrEP) for this population can likely lead to important reductions in HIV prevalence and incidence.

- Sub-Saharan African countries with limited resources such as Rwanda face a large HIV epidemic, so understanding how these strategies will affect the HIV epidemic is important.

What are the new findings?

- Continued efforts are needed to maintain consistent use of condoms and ART among FSWs in Rwanda

- Introduction of PrEP can also lead to improved control of the HIV epidemic in this country.

What do the new findings imply?

- This analysis confirms the need for a continued focus on FSWs in this country. Continuing to improve the quality and the access of existing preventive and treatment interventions with a new investment in PrEP will likely translate to the largest public health impact on the HIV epidemic.

as they have a high prevalence of HIV, engage in unprotected sex and have multiple partners. While there is some regional variation, the estimated HIV prevalence in the FSW population is 10 -fold to 20 -fold higher than in the general population. ${ }^{1}$ In Rwanda, the HIV prevalence among FSWs is estimated to be approximately $50 \% .^{2}$ In response, in the last two decades, Rwanda has developed a strong national response to control its HIV epidemic, including the FSW population. ${ }^{3}$ It has made impressive achievements in HIV care through progressive treatment guidelines 
and reduced the number of new HIV infections by $50 \%$ and the overall number of AIDS-related deaths by $78 \%$ from 2004 to $2014 .^{3}$ In Rwanda, the estimated number of new HIV infections in 2019 is 4409 (1846 male and 2563 female), and the estimated number of AIDS-related death in the same year is 2939 (1399 male and 1539 female). ${ }^{4}$ The national prevalence of HIV has been stable at $3 \%$ as of $2013 .{ }^{5}$ However, the country still faces challenges with high concentrations of HIV in the FSW population. Forty-six per cent of these women are estimated to be HIV positive ${ }^{6}$; HIV incidence among this population is 3.5 per 100 person-years (95\% CI 1.6 to 5.4), compared with the general population incidence rate $(0.27$ per 100 person-years, $95 \%$ CI 0.18 to 0.35$).{ }^{78}$ In Rwanda, it has been reported that only $25.0 \%$ (95\% CI $16.4 \%$ to $28.3 \%$ ) of FSWs have used condoms consistently in the last 30 days. ${ }^{6}$ Given that FSWs are more likely to have multiple sex clients too, interventions targeted for this high-risk group may yield important public health benefits.

Rwanda has specified efforts to test and treat HIV+ FSWs with antiretroviral therapy (ART) regardless of CD4 counts, in an effort to reduce HIV transmission. ${ }^{3}$ Targeted efforts include an intensive prevention package for FSWs. Mobile services and outreach activities have been implemented to reach FSW populations regardless of their location or work environment. ${ }^{3}$ At the facility level, services such as HIV counselling and testing, family planning and sexual reproductive health services, STI screening and treatment, and condom provision are offered to FSWs. ${ }^{3}$ Outreach involves hotel and bar-based services, street services and opportunities to engage FSWs involved in incidental transactions. ${ }^{3}$ Healthcare providers receive training to minimise stigma faced by FSWs and prevent avoidance for accessing healthcare services. ${ }^{3}$ In addition, implementation of pre-exposure prophylaxis (PrEP) targeted at FSWs is currently being considered for the next revision of HIV and AIDS National Strategic
Plan for the year 2019. This targeted intervention of PrEP along with the other efforts to increase utilisation of other HIV services and ART coverage for FSWs will likely result in reduced likelihood of HIV transmission.

It is therefore vital to understand how the incidence and prevalence of HIV will be affected by Rwanda's specific initiatives for FSWs. Given constrained resources for HIV treatment and prevention services, it is important to estimate the prevalence and incidence rates of HIV over time for strategic planning. Using nationally representative and FSW specific data in Rwanda, ${ }^{6-11}$ we developed a dynamic Markov model to estimate the prevalence and incidence rates of HIV among FSWs and sex clients over the next 10 years. In this study, we estimated the effects of ART, PrEP and condom-use-based prevention strategies on the HIV epidemic in Rwanda. In particular, we examine the evolution of incidence and prevalence in the FSW population, male sex clients and in the overall population.

\section{METHODS}

\section{Patient and public involvement}

Patients and the public were not directly involved in this study. However, the database used was developed with patients and public involvement (PPI) and is updated by a committee that includes patient representatives.

\section{Model structure}

We developed a nationwide dynamic Markov model ${ }^{12}$ for transmission of HIV from 2017 to 2027 to estimate the incidence and prevalence of HIV among FSWs, sex clients and the general population in Rwanda. Our model includes national level demographic data, sexual and sero-behavioural information of these three populations, HIV-associated morbidity, all-cause mortality and HIVrelated mortality. We considered the HIV transmission,

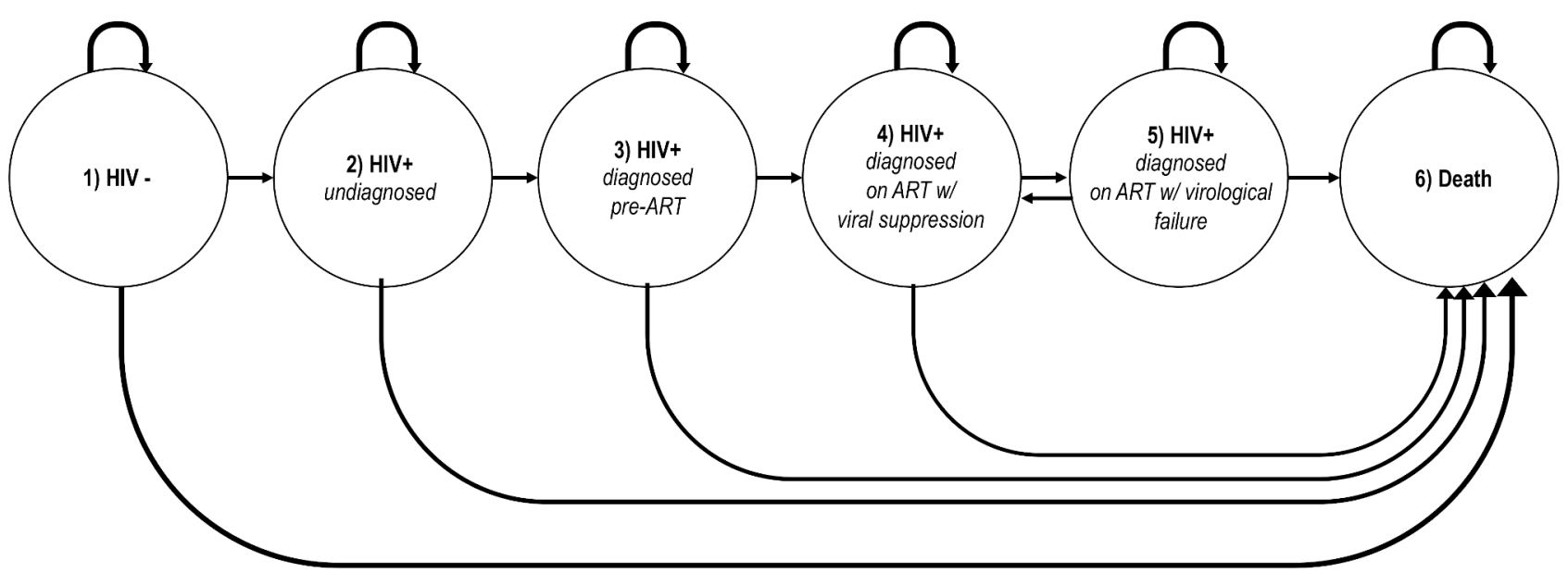

Figure 1 HIV transmission Markov model. For each underlying population, a Markov chain is considered with the state space, consisting of the six states: (1) HIV-; (2) HIV+ undiagnosed; (3) HIV+ diagnosed pre-ART; (4) HIV+ diagnosed on ART with viral suppression; (5) HIV+ diagnosed on ART with virological failure; and (6) death. State (5) represents a setting where viral suppression is not achieved under the current ART regimen, or where individuals discontinued ART for the duration of the 3-month cycle. ART, antiretroviral therapy; '+', positive; '-', negative. 
testing patterns, adherence to ART and their likelihood for viral suppression for FSWs, their male sex clients and the general population. Finally, as introduction of PrEP to FSWs is expected in Rwanda by year 2019, the model assumes and captures an expected 25\% PrEP uptake in the FSW population by the end of 2019 with a gradual increase to $65 \%$ PrEP use in the year 2027.

For each population, our Markov model considered the following six states: (1) HIV negative; (2) undiagnosed HIV+; (3) HIV+ pre ART; (4) HIV+ on ART with viral suppression; (5) HIV+ on ART without viral suppression; and 6) death. Here, state (5) represents a setting where viral suppression is not achieved under their ART regimen, or the individuals have discontinued ART for the duration of the cycle. Graphical representation of the Markov chain is depicted in figure 1. In addition, we considered transitions from one population to another, while remaining in the same state. That is, in each cycle, some transitions are accounted for from belonging to the general population and engaging in sex work or buying sex, and some FSWs and clients disengaging from having paid sex and moving back to the general population risk scheme for acquiring HIV. A time horizon of 10 years was considered starting in 2017 up to 2027. Cycle duration was set to 3 months with transition probabilities derived accordingly. Detailed description of our model is provided in the online supplementary material (see the Description of algorithm section).

\section{Model parameterisation}

Population sizes movement between populations

The sizes of the three considered populations were obtained from recent nationwide surveys and are presented in table 1 (individual calculations of these numbers are outlined in online supplementary table 1). The model also assumes an annual growth rate of $2.4 \%^{13}$ in Rwanda's population, as well as separate mortality rates for HIV negative individuals, undiagnosed or non-virally suppressed HIV+ individuals and virally suppressed HIV+ individuals on ART (see online supplementary table 2). Movement of individuals between the different population groups were considered as follows: We assumed that 47750 active FSWs reside in Rwanda and that, on average, FSW would remain active for 10 years before retiring; as a base case, that the number of FSWs would remain constant over the next 10 years (relative to country

Table 1 Sizes of the three populations and number of individuals within each state for each population

\begin{tabular}{|c|c|c|c|c|}
\hline Parameter & Base value & $\begin{array}{l}\text { Lower } \\
\text { bound }\end{array}$ & $\begin{array}{l}\text { Upper } \\
\text { bound }\end{array}$ & Source \\
\hline \multicolumn{5}{|l|}{ FSW population } \\
\hline HIV- (State 1) & 25964 & 13340 & 48950 & BBSS SW Rwanda $2015^{6}$ \\
\hline Undiagnosed HIV (State 2) & 983 & - & 53625 & BBSS SW Rwanda $2015^{6}$ \\
\hline Diagnosed HIV pre-ART (State 3) & 771 & - & 50734 & BBSS SW Rwanda $2015^{6}$ \\
\hline HIV+ with viral suppression on ART (State 4) & 9940 & 5444 & 35425 & BBSS SW Rwanda $2015^{6}$ \\
\hline $\begin{array}{l}\text { HIV+ without viral suppression on ART } \\
\text { (State 5) }\end{array}$ & 10092 & 1894 & 10266 & BBSS SW Rwanda $2015^{6}$ \\
\hline Total size & 47750 & 25000 & 111000 & ESPHS Rwanda $2011^{29}$ \\
\hline \multicolumn{5}{|l|}{ Sex client population } \\
\hline HIV- (State 1) & 133808 & 38070 & - & BBSS SW Rwanda $2015^{6}$ \\
\hline Undiagnosed HIV (State 2) & 4166 & 550 & - & BBSS SW Rwanda $2015^{6}$ \\
\hline Diagnosed HIV pre-ART (State 3) & 1859 & - & 31573 & UNAIDS $2016^{13} ;$ Nsanzimana $2015^{25}$ \\
\hline HIV+ with viral suppression on ART (State 4) & 9815 & 524 & - & UNAIDS $2016^{13}$; Nsanzimana $2015^{25}$ \\
\hline $\begin{array}{l}\text { HIV+ without viral suppression on ART } \\
\text { (State 5) }\end{array}$ & 6963 & 736 & - & UNAIDS $2016^{13}$ \\
\hline Total size & 156611 & 42300 & 177000 & ESPHS Rwanda $2011^{29}$ \\
\hline \multicolumn{5}{|l|}{ General population } \\
\hline HIV- (State 1) & 11467068 & 11395940 & 11502223 & NISR 2015; UNAIDS 2016 \\
\hline Undiagnosed HIV (State 2) & 24337 & - & 179973 & UNAIDS $2016^{13}$ \\
\hline Diagnosed HIV pre-ART (State 3) & 11874 & - & 231646 & UNAIDS $2016^{13}$; Nsanzimana $2015^{25}$ \\
\hline HIV+ with viral suppression on ART (State 4) & 156679 & 112499 & 244172 & UNAIDS $2016^{13} ;$ Nsanzimana $2015^{25}$ \\
\hline $\begin{array}{l}\text { HIV+ without viral suppression on ART } \\
\text { (State 5) }\end{array}$ & 161180 & - & 236966 & UNAIDS $2016^{13}$ \\
\hline Total size & 11809264 & - & - & NISR $2015^{5}$ \\
\hline
\end{tabular}

The base values used for the 2017-2027 model run were the output values for the calibrated model.

ART, antiretroviral therapy . 
population growth). Identical entry and exit rates were assumed for sex clients. For scenario analyses where the size of the FSW population was allowed to vary over time, the size of the sex client population was modified accordingly by holding number of sexual encounters and sex clients per FSW constant (the same as assumed for the base case) and mathematically solving for number of sex clients.

\section{HIV transmission among various populations}

We assumed transmission of HIV occurs between FSWs and their sex clients, as well as between the sex clients and the general population (eg, the male sex clients transmit the virus to their female sexual partners in the general population). In our analysis, we did not consider the case where FSWs could directly transmit HIV to the general population. We assumed an average number of 52 sex clients per year for each FSW, as well as an average number of 2.5 exposures (sex acts) per client, per year (ie, the average number of times a client seeks out an FSW annually). HIV transmission probabilities were computed using equations (1)-(4) provided in the online supplementary material (see the HIV transmission probabilities section of the supplementary material). In those equations, we incorporate the size of each population, HIV prevalence among the population of male sex clients, the average number of sexual exposures for each group, condom use or use of PrEP, the risk of HIV transmission per sexual intercourse (unprotected and protected) and the type of intercourse (anal vs vaginal). The values assumed for these parameters are presented and justified in online supplementary table 2.

The transition probabilities for the six states are presented in online supplementary table 3. In short, these probabilities were derived from multiple nationwide surveys and longitudinal studies conducted in Rwanda. In addition, general and Rwanda-specific estimates of likelihood of adherence to ART is incorporated. The model was programmed in R V.3.4.2, ${ }^{14}$ and figures were produced using the ggplot 2 package. ${ }^{15}$

\section{Model calibration}

We calibrated our model based on the rate of HIV prevalence among FSWs and the general population using data that were collected in $2010^{16}$ and $2015 .^{6}$ The estimated HIV prevalence among FSWs were $50 \%$ in 2010 and $45.5 \%$ in 2017 (online supplementary figure 1). We compared the estimated HIV incidence from our calibrated model with the HIV incidence rates that were reported by the UNAIDS's spectrum. ${ }^{417}$ The HIV prevalence among the sex clients was estimated to be $14.5 \%$ in 2015. The estimated national HIV prevalence has remained constant at approximately $3 \%$ over the past decade.

\section{Analysis}

Using the base values from the calibrated model, we recorded the evolution of annual HIV prevalence and
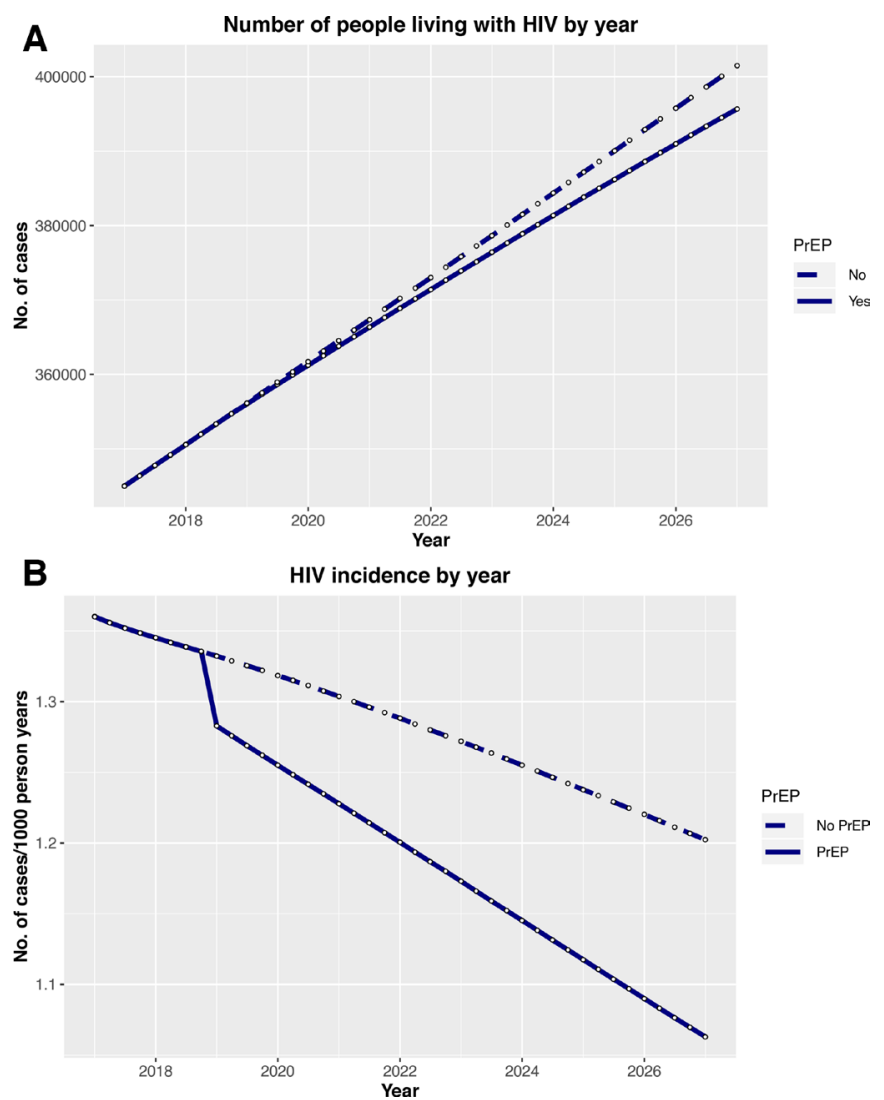

Figure 2 Effects of PrEP on the overall number of people living with HIV $(A)$ and the overall HIV incidence (B) over time. The effects of introducing PreP in 2019 (dotted lines) on the HIV prevalence and incidence for Rwanda as a nation are shown here. The solid line illustrates the estimated prevalence and incidence in Rwanda without the introduction of PrEP. PrEP, pre-exposure prophylaxis.

incidence from 2017 to 2027 in our Markov model. For each analysis, 1000 simulations with multinomial allocations of people to different Markov states each year were performed. Base case scenario input parameters are all presented in table 1 as well as in online supplementary tables 1 and 2 as described in the Methods section. We considered worst and best-case scenario analysis for key parameters related to testing behaviour and ART coverage, condom use and PrEP uptake, as well as growth or decline in the FSW population. The considered parameters and the assumed worst-case and best-case estimates are presented in online supplementary tables 4 and 5 .

\section{RESULTS}

\section{Base case 2027 projections}

The overall number of people living with HIV (ie, HIV prevalence) and the incidence rate of HIV (per 1000 person-years) from 2017 to 2027 in Rwanda are shown in figure 2. With PrEP, the number of people living with HIV increased from 344971 in 2017 to 395453 by year 2027 (figure 2A and online supplementary table 6), corresponding to a $1.37 \%$ annual growth (note, the general population growth rate is $2.41 \%$ ). With the current 

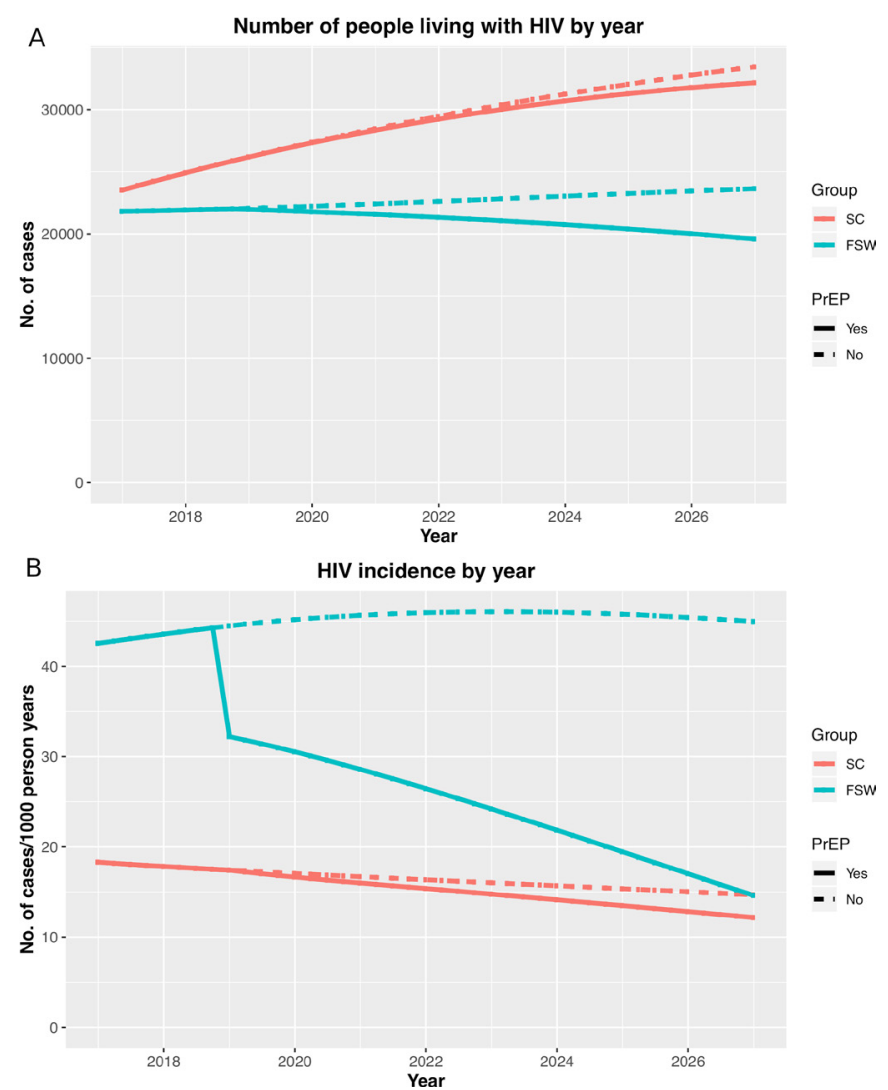

Figure 3 Effects of PrEP on the prevalence $(A)$ and incidence of HIV (B) for female sex workers and sex clients over time. The effects of introducing PreP in 2019 (dotted lines) on the HIV prevalence and incidence for SWs and SCs shown here. The solid line illustrates the estimated prevalence and incidence for these population without introduction of PrEP. PrEP, pre-exposure prophylaxis; SC, sex client; SW, female sex workers.

HIV testing and ART coverage, the annual incidence of FSWs living with HIV remained stable over the 10-year period. With the current HIV testing and ART coverage, the annual overall population incidence rate decreased from 2017 (1.36 per 1000 person-years) to 2027 (1.20 per 1000 person-years) (online supplementary table 7 ). With the introduction of PrEP in 2019, the annual incidence decreased further with a notable additional decrease in 2020, and a continuing decrement to 1.06 per 1000 person-years in 2027 (figure 2B).

With the introduction of PrEP, the number of HIV positive FSWs decreased from 21818 in 2017 to 19511 in 2027 (figure 3A and online supplementary table 8), and the incidence among FSWs decreases from 42.59 per 1000 person-years in year 2017 to 14.65 per 1000 person-years in 2027 (figure 3B). Among sex clients, the number of sex clients infected with HIV increased from 23538 to 32104 , corresponding to an annual growth of $3.15 \%$ (ie, faster than the annual overall population growth); the HIV incidence decreased from 18.30 per 1000 person-years in 2017 to 12.11 per 1000 person-years in 2027 (online supplementary table 9). The evolution of the number and proportion of FSWs and sex clients living with HIV in the base case scenario are presented in online supplementary figures 2 and 3 . Overall evolution of proportion of FSWs and sex clients in each of the six states for the base case scenario are presented in online supplementary figure 4 .

\section{Scenario analysis}

Online supplementary table 4 presents the worst-case and best-case scenarios for the 2027 HIV prevalence estimates for the selected scenario analysis parameters. The tornado diagrams illustrating each of the three populations' sensitivities to changes in the explored parameters (ie, magnitude of change in results under the worst-case and best-case scenarios) are provided in online supplementary figure 5 . For the parameter of testing and treatment, we observed the following trends. Increased testing for FSWs was associated with a small reduction in the HIV prevalence among sex clients. Increased testing of the general population combined with increased national coverage of ART were associated with a reduction in the HIV prevalence among FSWs and their male sex clients. Improved adherence to ART had a moderate impact on the HIV prevalence in FSWs, their male sex clients and the general population, but a worsening in ART adherence was associated with a larger negative effect with the increased HIV prevalence. Changes in HIV testing or ART-related parameters, however, never exceeded $1.1 \%$. For use of condoms, the best-case scenario for increased use among FSWs reduces the prevalence by $7.86 \%$ $(37.37 \%$ vs $29.51 \%)$ among the FSW population and by $5.97 \%$ (17.87\% vs $11.90 \%)$ among their male sex clients. In the worst-case scenario with decreased condom use among FSWs, however, the models estimated a $4.59 \%$ and $3.4 \%$ increase in the HIV prevalence in FSWs and their male sex clients, respectively. An increase in condom use among FSWs reduced the HIV prevalence in the overall population by $0.11 \%$. The best-case scenario of increased condom use in the general population yields a $0.17 \%$ absolute decrease in the HIV prevalence in the overall population (ie, a $6.46 \%$ relative reduction) but did not affect the prevalence among FSWs or their male sex clients.

Similarly, a worst-case reduction in condom use in the general population was associated with an increase in the HIV prevalence. The best-case scenarios for PrEP use among FSWs resulted in a $1.28 \%$ reduction in the HIV prevalence among FSWs, whereas in the worst-case scenario, a $1.27 \%$ increase in the HIV prevalence can be expected. Best-case scenarios for condom use and PrEP use yielded the largest reduction in the HIV prevalence overall, and no further reduction were observed from added HIV testing, ART coverage and adherence under this scenario. However, the worst-case scenarios for condom use and PrEP were still worsened further, if combined with worst case scenarios for HIV testing, ART coverage and adherence.

The scenario analyses and the tornado diagrams of the 2027 HIV incidence estimates (per 1000 person-years) 
are presented in online supplementary table 5 and figure 6 , respectively. Different scenario analyses showed incidence estimates that aligned similar to the prevalence estimates. Changes in testing and treatment for ART showed small changes in the HIV incidence rates for FSWs, their male sex clients and the general population; collectively, when testing for FSWs and general population was combined with increased ART coverage and adherence, the estimated HIV incidence for sex clients showed large reduction (Scenario 1E: 12.45 to 11.29 cases per 1000 person-years). In the best-case scenario with increased condom use among FSWs, the models estimated a decrease in the HIV incidence by 12.25 cases per 1000 person-years (down to 3.59 per 1000 person-years) for FSWs and 0.16 cases per 1000 person-years (down to 0.92 per 1000 person-years) for the overall population, but increased condom use in FSWs did not affect the HIV incidence rates for the general population. The best-case scenarios for PrEP use among FSWs resulted in a $27.46 \%$ relative reduction in incidence among FSWs. Best-case scenarios for condom use combined with PrEP use yielded the largest reduction in the incidence among the FSWs, sex clients and the overall population, and no important reductions were observed from the best-case scenarios of improved HIV testing, ART coverage and ART adherence.

\section{DISCUSSION}

Since 2016 after implementing the test-and-treat strategy, Rwanda has observed drastic improvements in several HIV-related outcomes. ${ }^{8}$ The national ART coverage and testing has increased considerably in the last decade. The overall number of people who are now aware of their HIV status and treated with ART has also improved. In combination, these measures have led to a stabilisation of the overall HIV prevalence at $3 \%$ in Rwanda. The continued epidemic of HIV in Rwanda is largely driven by the concentrated HIV epidemic among FSWs and their sex clients. Our findings highlight a number of key components for containing and reducing the HIV epidemic in Rwanda. A continued promotion of ART-based prevention strategies, particularly among high-risk population such as FSWs, is essential in keeping the HIV epidemic stabilised in this country. While having a minor effect in the general population, the expected introduction of PrEP will likely have a substantial impact on both the incidence and prevalence of FSWs and sex clients, with the HIV incidence among FSWs greatly being reduced by PrEP.

There have been other FSW economic models that have previously been published (table 2) ${ }^{18-23}$ To our knowledge, our Markov model is the first to examine effects of the ART, condoms and PrEP, specifically, in the geographical setting of Rwanda. The focus of other studies has been on regions such as Côte d'Ivoire, India, Benin and South Africa, with a deterministic modelling approach for some. Similar to our study, the majority of these models $(n=4 / 6)$ considered three populations in FSWs, sex clients and the general population. The economic model by Maheu-Giroux et $a l^{18}$ considered another high-risk group in men who have sex with other men. The model by Mukandavire $e t a l^{19}$ considered the role of pimps in the HIV epidemic, but this model was not specific to any countries. Mukandavire $e t a l^{19}$ was also the only other economic model that considered the role of PrEP and condoms on the HIV prevalence and incidence; however, this study did not consider the role of ART simultaneously with these other two preventive intervention strategies.

Our model and analyses have several strengths and limitations. Our model capitalised on access to rich up-to-date nationwide Rwandan-specific data sources and interaction with the Ministry of Health officials who were available to provide the local context. As a result, most of the transition probabilities are directly informed by data with high internal validity. ${ }^{12}$ The used data, however, are all cross-sectional and not representative of the local subpopulations of FSWs that may have different geographical challenges and constraints. In addition, it is possible that the estimated value may be under-reported, particularly for sexual risk behaviour questions (eg, number of sexual partners) that were selfreported. There was limited information on behaviours of sex clients, and as a result, it was necessary to indirectly model these via the links to FSWs and the general population. To estimate the HIV transmission probabilities for our Markov model, we had to rely on self-reported data on sexual behaviours, which are likely subject to some bias. Our model did not include other key risk populations (eg, men who have sex with men, male sex workers and sero-discordant couples) in Rwanda.

As Rwanda prepares to plan for its fourth edition of its National Strategy Plan for HIV/AIDS, it recognises the necessity for a continued focus on FSWs. Efforts to increase the testing of HIV in FSWs, ensuring lifelong ART in HIV positive FSWs with provision at diagnosis, and education of this group, sex clients and other members of the general population through public health education and health promotion campaigns will need to be continued, along with efforts to improve consistent condom use. Our model shows that improving the condom use will have a substantial effect on HIV prevalence in the FSW and general population. Promoting condom use among FSWs will dramatically reduce prevalence among sex clients over the next 10 years. Moreover, the planned introduction of PrEP to FSWs in 2019 will have a substantial effect on the FSW population as the incidence of HIV drops drastically after its (modelled) introduction and continues to decline steeply. The individual prophylactic efficacy of increased testing and ART coverage, condom use and PrEP uptake among HIV negative FSWs hold much promise, and in the long run, may be the longterm strategy to control the HIV epidemic in Rwanda.

Continuing to improve the quality of and access to existing prevention and treatment interventions along 


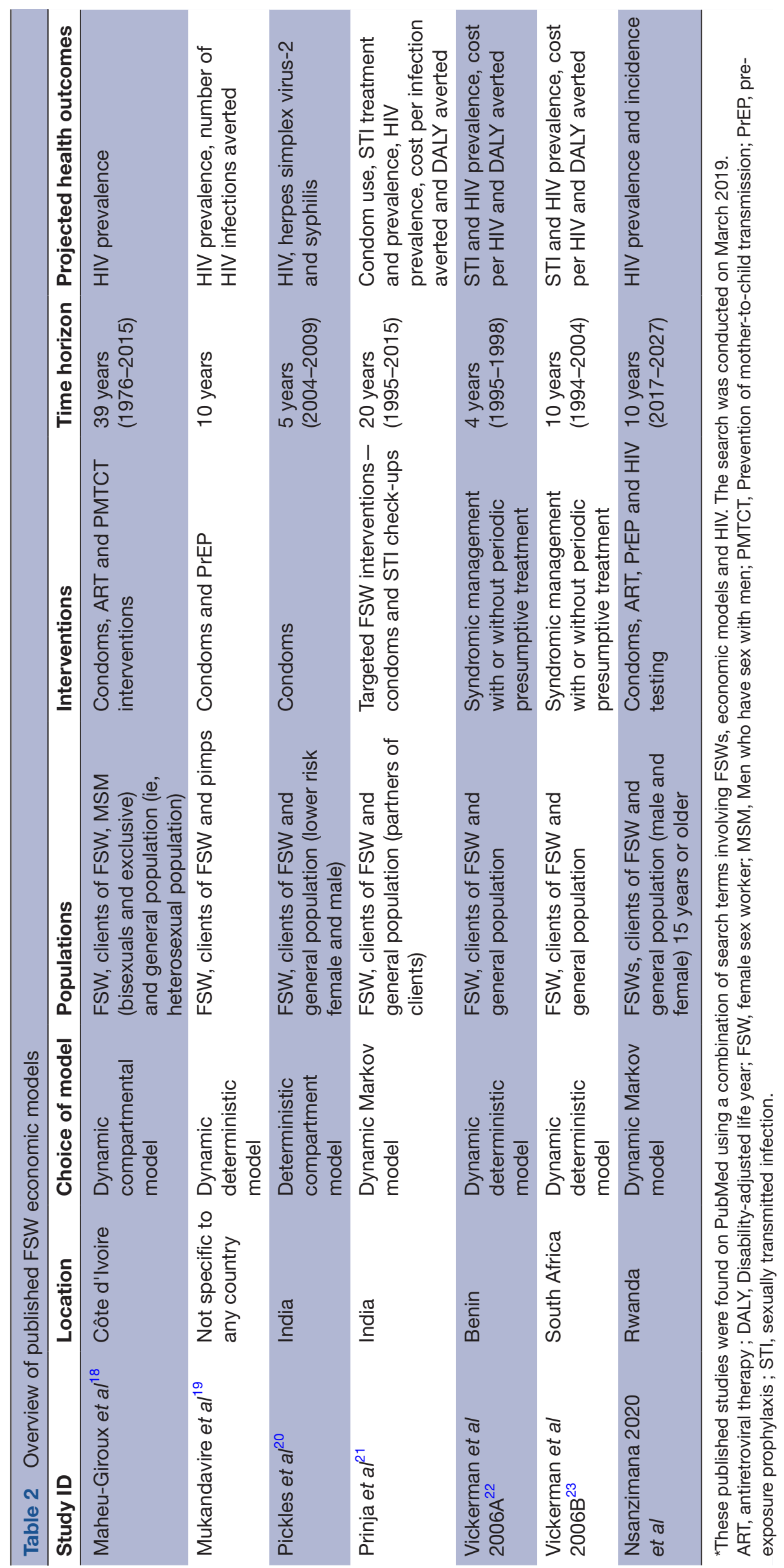


with the new investment in PrEP in FSWs will likely have the largest public health impact on the HIV epidemic. While HIV care in Rwanda has been among the best in sub-Saharan Africa in terms of expanding ART coverage through investments in HIV infrastructure, ${ }^{24} 25$ it will require tremendous continued efforts. Introducing PrEP will require more resources, and this needs to be complemented and supported with other aspects of the HIV treatment cascade. Improving access and adherence to ART and condom use, along with achieving $65 \%$ coverage of PrEP, is ambitious an target. Such efforts will require multisector response and coordinated participation and actions across all groups of stakeholders in Rwanda. Healthcare providers will need to receive continued training to minimise stigma and discrimination faced by FSWs in order to ensure that they will not avoid accessing key healthcare services such as regular HIV testing, PrEP and ART. ${ }^{26}$ As well, economic empowerment and opportunities with educational support are also likely required for this key population to control the concentrated HIV epidemic. $^{12728}$

Despite the progress made in containing the HIV epidemic in Rwanda, the concentration of the HIV epidemic remains high in marginalised groups such as FSWs. There have been limited investigations on FSWs. Future research that needs to be prioritised include FSWs' ability to negotiate condom use with their clients and ways to increase diagnostic testing of HIV and other STIs in FSWs. Existing barriers that affect FSWs' acceptability and adherence to ART and PrEP should be explored and addressed to remove barriers that are institutionalised. This study has demonstrated the need for continued efforts to improve and expand the quality of existing HIV services (eg, more testing and ART), as well the potential gain of adopting a targeted intervention strategy such as PrEP in FSWs and other key drivers of the HIV epidemic not only in Rwanda but also in other sub-Saharan African countries.

\section{Author affiliations \\ ${ }^{1}$ Rwanda Biomedical Center, Kigali, Rwanda \\ ${ }^{2}$ Basel Institute for Clinical Epidemiology and Biostatistics, University of Basel, Basel, Switzerland \\ ${ }^{3}$ Department of Health Research Methods, Evidence, and Impact, McMaster University, Hamilton, Canada, McMaster University, Hamilton, Ontario, Canada ${ }^{4}$ Real World and Advanced Analytics, Cytel Inc, Vancouver, BC, Canada ${ }^{5}$ Institute for HIV, Diseases Prevention and Control, Rwanda Biomedical Center, Kigali, Rwanda \\ ${ }^{6}$ School of Medicine and Hubert Department of Global Health, Rollins School of Public Health, Emory University, Atlanta, United States \\ ${ }^{7}$ School of Medicine and Pharmacy, Department of Primary Health Care, University of Rwanda, Kigali, Rwanda \\ ${ }^{8}$ Department of Public Health, Center for Global Health, Aarhus University, Aarhus University, Aarhus, Denmark \\ ${ }^{9}$ Experimental Medicine, Department of Medicine, University of British Columbia, Vancouver, BC, Canada \\ ${ }^{10}$ School of Public Health, University of Rwanda, Kigali, Rwanda}

Acknowledgements This paper was based from SN's PhD thesis titled, "Linkage to and retention in HIV care and treatment in the Rwanda national HIV program", which is hosted by the University of Basel repository (https://edoc.unibas.ch/ 67838/).
Contributors SN, EJM, OH, JJHP, LD, HCB and KT contributed to the conception and design of the work. SN, EJM, OH, JJHP and KT contributed to data analysis. SN, EJM, OH, PM, EK, JPU, JJHP, LD, JC, HCB and KT contributed to the data interpretation, drafting the article, critical revision of the article and final approval of the text.

Funding This study was funded by the Rwanda Biomedical CenterCentre (RBC).

Disclaimer The RBC did not have any role in the study design, collection, analysis and interpretation of the data.

\section{Competing interests None declared.}

Patient and public involvement Patients and/or the public were not involved in the design, or conduct, or reporting or dissemination plans of this research.

Patient consent for publication Not required.

Provenance and peer review Not commissioned; externally peer reviewed.

Data availability statement All data relevant to the study are included in the article or uploaded as supplementary information.

Open access This is an open access article distributed in accordance with the Creative Commons Attribution Non Commercial (CC BY-NC 4.0) license, which permits others to distribute, remix, adapt, build upon this work non-commercially, and license their derivative works on different terms, provided the original work is properly cited, appropriate credit is given, any changes made indicated, and the use is non-commercial. See: http://creativecommons.org/licenses/by-nc/4.0/.

\section{ORCID iD}

Edward J Mills http://orcid.org/0000-0003-3120-9694

\section{REFERENCES}

1 World Health Organization. Preventing HIV in sex work settings in sub-Saharan Africa. Geneva, Switzerland: WHO, 2011

2 Ngugi EN, Roth E, Mastin T, et al. Female sex workers in Africa: epidemiology overview, data gaps, ways forward. Sahara J 2012;9:148-53.

3 Republic of Rwanda, Ministry of Health. Rwanda HIV and AIDS National Strategic Plan July 2013 - June 2018. Rwanda Biomedical Center, 2014.

4 UNAIDS. National HIV estimates file: UNAIDS. Available: https:// www.unaids.org/en/dataanalysis/datatools/spectrum-epp [Accessed Feb 15 2019].

5 National Institute of Statistics of Rwanda (NISR) [Rwanda], Ministry of Health (MOH) [Rwanda], and ICF International. Rwanda demographic and health survey 2014-15. Rockville, Maryland, USA: $\mathrm{NISR}, \mathrm{MOH}$, and ICF International, 2015.

6 Institute of HIV/AIDS Disease Prevention and Control HIV-AIDS,, STIs \& Other Blood Borne Infections Division. The Behavioral \& Biological Surveillance Survey Among Female Sex Workers in Rwanda in 2015. Rwanda Biomedical Center, 2016.

7 Braunstein SL, Ingabire CM, Kestelyn E, et al. High human immunodeficiency virus incidence in a cohort of Rwandan female sex workers. Sex Transm Dis 2011;38:385-94.

8 Nsanzimana S, Remera E, Kanters S, et al. Household survey of HIV incidence in Rwanda: a national observational cohort study. Lancet HIV 2017;4:e457-64.

9 Nsanzimana S, Remera E, Kanters S, et al. Effect of baseline CD4 cell count at linkage to HIV care and at initiation of antiretroviral therapy on mortality in HIV-positive adult patients in Rwanda: a nationwide cohort study. Lancet HIV 2015;2:e376-84.

10 Nsanzimana S, Remera E, Kanters S, et al. Life expectancy among HIV-positive patients in Rwanda: a retrospective observational cohort study. Lancet Glob Health 2015;3:e169-77.

11 Mutagoma M, Kayitesi C, Gwiza A, et al. Estimation of the size of the female sex worker population in Rwanda using three different methods. Int J STD AIDS 2015;26:810-4.

12 Briggs AH, Claxton K, Sculpher MJ. Decision modelling for health economic evaluation: handbooks in health economic E, 2006.

13 UNAIDS. Country factsheets Rwanda, 2016. Available: http://www. unaids.org/en/regionscountries/countries/rwanda [Accessed 31 Oct 2017].

$14 \mathrm{R}$ Core Team. R: A language and environment for statistical computing (v.3.4.2) [program]. Vienna, Austria: R Foundation for Statistical Computing, 2017.

15 Wickham H, Chang W. ggplot2: an implementation of the grammar of graphics. R package version 07, 2008. 
16 Republic of Rwanda, Ministry of Health. Behavioral \& biological surveillance survey among female sex workers, Rwanda - 2010 survey report. Rwanda Biomedical Center, 2010.

17 Stover J, Brown T, Puckett R, et al. Updates to the Spectrum/ Estimations and projections package model for estimating trends and current values for key HIV indicators. AIDS 2017;31(Suppl 1):S5-11.

18 Maheu-Giroux M, Vesga JF, Diabaté S, et al. Changing dynamics of HIV transmission in Côte d'Ivoire: modeling who acquired and transmitted infections and estimating the impact of past HIV interventions (19762015). J Acquir Immune Defic Syndr 2017;75:517-27.

19 Mukandavire Z, Mitchell KM, Vickerman P. Comparing the impact of increasing condom use or HIV pre-exposure prophylaxis (PrEP) use among female sex workers. Epidemics 2016;14:62-70.

20 Pickles M, Foss AM, Vickerman P, et al. Interim modelling analysis to validate reported increases in condom use and assess HIV infections averted among female sex workers and clients in southern India following a targeted HIV prevention programme. Sex Transm Infect 2010;86(Suppl 1):i33-43.

21 Prinja S, Bahuguna P, Rudra S, et al. Cost effectiveness of targeted HIV prevention interventions for female sex workers in India. Sex Transm Infect 2011;87:354-61.

22 Vickerman P, Watts C, Peeling RW, et al. Modelling the cost effectiveness of rapid point of care diagnostic tests for the control of HIV and other sexually transmitted infections among female sex workers. Sex Transm Infect 2006;82:403-12.
23 Vickerman P, Terris-Prestholt F, Delany S, et al. Are targeted HIV prevention activities cost-effective in high prevalence settings? Results from a sexually transmitted infection treatment project for sex workers in Johannesburg, South Africa. Sex Transm Dis 2006;33:S122-32.

24 Binagwaho A, Farmer PE, Nsanzimana S, et al. Rwanda 20 years on: investing in life. Lancet 2014;384:371-5.

25 Nsanzimana S, Kanters S, Remera E, et al. HIV care continuum in Rwanda: a cross-sectional analysis of the National programme. Lancet HIV 2015;2:e208-15.

26 Kouta $\mathrm{C}$, Pithara $\mathrm{C}$, Zobnina A, et al. A systematic review of training interventions addressing sexual violence against marginalized at-risk groups of women. Health Educ Res 2015;30:971-84.

27 Kim J, Pronyk P, Barnett T, et al. Exploring the role of economic empowerment in HIV prevention. AIDS 2008;22 Suppl 4:S57-71.

28 Kennedy CE, Fonner VA, O'Reilly KR, et al. A systematic review of income generation interventions, including microfinance and vocational skills training, for HIV prevention. AIDS Care 2014;26:659-73.

29 Rwanda Biomedical Center/Institute of HIV/AIDS, Disease Prevention and Control Department (RBC/IHDPC), School of Public Health (SPH), UNAIDS, and ICF International. Estimating the size of populations through a household survey. Calverton, Maryland, USA: RBC/IHDPC, SPF, UNAIDS, and ICF International, 2012. 